\title{
Practical Tips
}

\section{Medically Unlikely Edits}

\author{
By Julia E. Tomkins
}

American Society of Clinical Oncology, Alexandria, VA

Many oncology providers and their staff are asking the questions, "Why are we receiving denials on the amount of drug billed?" and "Why is Medicare limiting the amount of drug reported?" The answers relate to Medicare's medically unlikely edits, commonly referred to as MUEs. MUEs are not new, as the Centers for Medicare \& Medicaid Services (CMS) created and implemented the edits a number of years ago on Current Procedural Terminology (CPT) codes. Providers have, however, been seeing an increase in the number of denials relating to MUEs on Healthcare Common Procedure Coding System (HCPCS) codes. This article will provide insight on these edits, provide guidance on reporting and commenting on MUEs, and describe the impact on oncology practice.

MUEs differ from the National Correct Coding Initiative (NCCI) edits. The NCCI edits identify which procedures and services are bundled and should not be billed together. MUEs limit the number of times a service or procedure can be reported by a physician on the same date of service to an individual patient. MUEs apply not only to services and procedures but also to durable medical equipment and drugs. CMS developed the MUE program to reduce the error rate for Part B coding and to control improper payments. The edits are based on anatomic considerations, HCPCS/CPT code descriptors, CPT coding instruction, CMS policies, nature of service or procedure, and nature of analyte or equipment. Generally speaking, the MUEs are considered a tool for limiting fraud and abuse activity, which has become a focus of the agency over the past few years.

CMS has established a process for developing MUEs. Once CMS determines a limit on a specific code, the proposed edit is shared with select entities such as the American Medical Association, national medical societies, and other national health care organizations for comment. (ASCO is one of the national specialty societies allowed to review and comment on the proposed edits.) The proposed MUEs and CMS's responses to comments submitted on the proposed edits are considered strictly confidential and cannot be shared. Final decisions regarding the MUEs ultimately lie with CMS. MUEs are updated on a quarterly basis and many are published by CMS on their Web site. It is important to note that although a large number of the edits are available on the agency's Web site, some MUEs are considered confidential and are not released.

Limits established through the MUE program are line itemspecific, and the edits are adjudicated on each line of a claim. Therefore, if the unit of service for a particular procedure or drug per claim line item exceeds the established limit, that line item will be denied. For purely illustrative purposes, imagine the MUE for J7040 - infusion, normal saline solution, sterile-is 6 . If a provider reports the units of service as 8 on the line item for J7040, the line item would be denied because it exceeds the MUE of 6 units.

CMS has published a number of frequently asked questions (FAQS) related to the MUE program, and those can be found on the agency's Web site http://www.cms.gov/NationalCorrect CodInitEd/08_MUE.asp\#TopOfPage. In those FAQs, CMS states that as of April 1, 2010, claim line denials based on MUEs may be appealed to local Medicare contractors. (Before April 1, 2010, claims were returned to providers rather than denied and there was no appeals process applicable.) The agency also describes how medically reasonable and necessary units of service in excess of a MUE can be reported. The following reporting instructions are provided by CMS:

"Since each line of a claim is adjudicated separately against the MUE value for the code on that line, the appropriate use of CPT modifiers to report the same code on separate lines of a claim will enable a provider/supplier to report medically reasonable and necessary units of service in excess of an MUE value. CPT modifiers such as -76 (repeat procedure by same physician), -77 (repeat procedure by another physician), anatomic modifiers (eg, RT, LT, F1, F2), -91 (repeat clinical diagnostic laboratory test), and -59 (distinct procedural service) will accomplish this purpose. Modifier -59 should be utilized only if no other modifier describes the service."

A change to an MUE can be requested in writing to the CMS contractor responsible for implementing and maintaining the MUE program, Correct Coding Solutions, LLC. In its FAQs, CMS states that it is "generally recommended that individuals contact their national healthcare organization before writing to Correct Coding Solutions, LLC.” CMS also notes that the national organization's support and assistance may be helpful in requesting a change to an established MUE value.

As mentioned, oncology providers have been seeing an increase in line item denials for certain drugs. Because the MUEs for most drugs are not currently published by CMS, one must either refer to the denial code/reason or contact the local Medicare contractor to confirm the denial was based on a MUE. Denials based on MUEs may create a number of challenges and burdens to an oncology practice. Jeffery Ward, MD, of Puget Sound Cancer Center and a member of ASCO's Clinical Practice Steering Subcommittee, sums up the impact of MUEs in oncology this way, "Besides the very significant administrative 
costs associated with inappropriate MUEs are the impact they have on the patient and their ability to trust their physician, clinic, and Medicare to appropriately meet needs during a time that they feel particularly vulnerable and buffeted by a most frightening disease." Below are some of the burdens practices are facing:

- Administrative burden: This includes the cost of staff researching and/or confirming the denial, identifying the appropriate action, and completing the refiling or reopening of claims.

- Financial challenges: These stem from not being reimbursed for medically necessary services provided to patients. Chemotherapy drugs tend to be more costly than some other categories of drugs.

- Loss of productivity: This includes the loss of office staff time working to identify denied line items and resubmit, nursing time verifying services provided, and physician's time in educating about coding edits/denied services.

ASCO reviewed a number of MUEs and raised concerns with CMS regarding MUEs applied to chemotherapy (and supportive care) drugs. As a result of CMS's efforts to limit fraudulent and abusive activity, limits will continue to be applied to oncology-related drugs. As mentioned previously, CMS makes all final decisions related to the limits placed on CPT and HCPCS codes.

ASCO has been successful in requesting adjustments to some of the MUEs on drugs. Because of the variety of accepted and recognized chemotherapy treatments and regimens (which may use drugs that are FDA approved for the indication or that fall under off-label use) and the varying clinical circumstances of each individual cancer patient, it is challenging to identify one limit that will work for every patient, every time. ASCO will continue to work with Correct Coding Solutions and CMS to appropriately adjust edits. If you are receiving denials for CPT/HCPCS codes based on MUEs, you can contact ASCO's Practice Division of the Cancer Policy and Clinical Affairs Department by sending an e-mail to practice@asco.org.

Accepted for publication on March 28, 2011.

Author's Disclosures of Potential Conflicts of Interest The author indicated no potential conflicts of interest.

Corresonding author: Julia E. Tomkins, ASCO, 2318 Mill Rd, Suite 800, Alexandria,VA 22314; e-mail: julia.tomkins@asco.org.

DOI: 10.1200/JOP.2011.000286

\section{TAKE ADVANTAGE OF ASCO'S EXCLUSIVE MEMBER-ONLY BENEFITS}

Are you experiencing the full value of ASCO membership? Along with deep discounts on meeting registrations and premier oncology publications like Journal of Clinical Oncology, ASCO also has a host of member-only benefits.

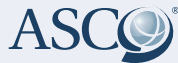

American Society of Clinical Oncology 